\title{
ON ISOMORPHISMS AND HYPER-REFLEXIVITY OF CLOSED SUBSPACE LATTICES
}

\author{
HAN DEGUANG \\ Department of Mathematics \\ Qufu Normal University, Shandong \\ P.R. China
}

(Received March 15, 1988 and in revised form January 2, 1991)

\begin{abstract}
There are some papers, such as [1], [2] and [3], in which some properties on isomorphism of closed subspace lattices of Hilbert spaces were studied. In this short paper we will point out that the hyper-reflexivity of closed subspace lattice is invariant under isomorphism of $\xi\left(H_{1}\right)$ on $\xi\left(H_{2}\right)$. We also proved that if $T$ is in $L(H)$ such that $0 \bar{\epsilon} \pi(T)$ and $\mathscr{F}$ is a hyper-reflexive subspace lattice, then $\phi_{T}(\mathscr{F}) \cup\{H\}$ is hyper-reflexive where $\phi_{T}$ is a homomorphism induced by $T$.
\end{abstract}

KEY WORDS AND PHRASES: Reflexivity, Hyper-reflexivity, Isomorphism.

1980 AMS SUBJECT CLASSIFICATION CODES. 46C10, 47D25.

\section{INTRODUCTION.}

Let $H$ be a complex Hilbert space, $L(H)$ denotes the set of all bounded linear operators on $H$ and let $\xi(H)$ be the set of all closed subspaces of $H$. For any subset $\mathcal{A}$ of $L(H)$ and any family $\mathscr{F} \subseteq(H)$, let $\mathcal{A}$ denote the lattice of closed subspaces invariant for $\mathcal{A}$ (or the lattice of invariant projections for $\mathcal{A}$ ) and let $\mathrm{Alg} \mathcal{F}$ be the set of all operators invariant for $\mathscr{F}$. $\mathscr{F}$ is said to be reflexive if Lat Alg $\mathscr{F}=\mathscr{F}$. A subalgebra $\mathcal{A}$ of $L(H)$ is said to be reflexive if $\mathcal{A}=\operatorname{Alg}$ Lat $\mathcal{A}$.

Let $\mathcal{A}$ be a reflexive algebra and let $T \in L(H)$. It is easy to see that

$$
\operatorname{dist}(T, \mathcal{A}) \geq \sup \left\{\left\|P^{\perp} T P\right\|: P \in \text { Lat } \mathcal{A}\right\}
$$

$\mathcal{A}$ is called to be hyper-reflexive (See [4]) if there exists a constant $K>0$ such that for any $T \in L(H)$

$$
\operatorname{dist}(T, \mathcal{A}) \leq \mathrm{K} \sup \left\{\left\|P^{\perp} T P\right\|: P \in \text { Lat } \mathcal{A}\right\}
$$

For any subspace lattice $\mathscr{F} \subseteq \xi(H)$, if $\mathscr{F}$ is reflexive and Alg $\mathscr{F}$ is hyper-reflexive, then $\mathscr{F}$ is said to be hyper-reflexive. Let $\phi$ be a lattice isomorphism of $\xi\left(H_{1}\right)$ onto $\xi\left(H_{2}\right)$ (i.e., a bijection with the 
property that $M=N$ iff $\phi(M)=\phi(N))$. It was proved in [1] and [2] that $\mathscr{F}$ is reflexive if and only if $\phi(F)$ is reflexive. For an operator $A \in L(H), A$ can give rise to a map $\phi_{A}: \xi(H) \rightarrow \xi(H)$ given by $\phi_{A}(M)=\overline{A M}$, where ' $-'$ denotes norm closure. In the paper [3] the author proved that if the approximate point spectrum $\pi(A)$ of $A$ does not contain 0 and $\mathscr{\sigma}$ is reflexive, then $\phi_{A}(\mathscr{F}) \cup\{H\}$ is also reflexive. Here we prove the following theorem:

LEMMA 1. Let $H_{1}$ and $H_{2}$ be two complex Hilbert spaces, and let $\phi$ be a lattice isomorphism of $\xi\left(H_{1}\right)$ onto $\xi\left(H_{2}\right)$. Then a subspace lattice $\mathscr{F}$ of $\xi\left(H_{1}\right)$ is hyper-reflexive if and only if $\phi(\mathscr{F})$ is hyper-reflexive.

THEOREM 2. Let $H$ be a complex Hilbert space, $A \in L(H)$ and $0 \bar{\epsilon} \pi(A)$. If the subspace lattice $\mathscr{F}$ of $\xi(H)$ is hyper-reflexive, then so is $\phi_{A}(\Im) U\{H\}$.

2. THE PROOF OF THE THEOREMS.

Lemma 1 may be known, we give a proof by the following theorem:

THEOREM A. ([4]) Let $\mathcal{A} \subseteq L(H)$ be a $\sigma$-weakly closed unital subalgebra of $L(H)$. Then $\mathcal{A}$ is hyper-reflexive iff every element $f \in \mathcal{A}_{\perp}$ has a representation

$$
f=\sum_{n=1}^{\infty} f_{n}
$$

where $\mathcal{A}_{\perp}=\{f: f$ is a $\sigma$-weakly continuous linear functional on $L(H)$ and $f(\mathcal{A})=\{0\}\}$, each $f_{n}$ is an elementary functional in $\mathcal{A}_{\perp}$ and $\sum_{n=1}^{\infty}\left\|f_{n}\right\|<\infty$.

REMARK. A $\sigma$-weakly continuous functional $f$ on $L(H)$ is said to be elementary if there exist $x, y \in H$ suc that $f(T)=(T x, y)$ for any $T \in L(H)$. We write $f=(x \otimes y)$.

Let $S$ be a conjugate linear continuous map from $H_{1}$, into $H_{2}$. It can be defined uniquely the adjoint $S^{*}$ of $S$ by the formula

$$
\left(S^{*} x, y\right)=(S y, x)=\overline{(x, S y)}
$$

It is easy to check that $\left(S^{*}\right)^{*}=S$, and $\left(S^{-1}\right)^{*}=\left(S^{*}\right)^{-1}$ when $S$ has continuous inverse.

PROOF OF LEMMA 1. It is sufficient to prove the necessity. First $\phi(\mathcal{F})$ is reflexive by the reflexivity of $\mathcal{F}$. If $\operatorname{dim} H_{1}<\infty$, it is easy to prove that $\operatorname{Alg} \phi(\mathcal{F})$ is hyper-reflexive ([5]). Now suppose that $\operatorname{dim} H_{1}=\infty$. Then there exists a bicontinuous linear or conjugate linear bijection $S: H_{1} \rightarrow H_{2}$ such that $\phi=\phi_{S}$ i.e., $\phi(M)=S M$ for every $M \in \xi\left(H_{1}\right)$ (see [1]).

We may suppose that $S$ is conjugate linear. For any $f \in(\operatorname{Alg} \phi(\mathcal{F}))_{\perp}$, define $g(A)=\overline{f\left(S A S^{-1}\right)}$, then $g \in(\mathrm{Alg} \mathscr{F}) \perp$ since $\mathrm{Alg} \phi(\mathscr{F})=S(\mathrm{Alg} \mathscr{F}) S^{-1}$.

By theorem $A$, there exist $x_{n}, y_{n} \in H_{1}$ such that $\left(x_{n} \otimes y_{n}\right) \in($ Alg $\mathscr{F}){ }_{\perp}$,

$$
\begin{gathered}
g=\sum_{n=1}^{\infty}\left(x_{n} \otimes y_{n}\right) \\
\sum_{n=1}^{\infty}\left\|x_{n}\right\|\left\|y_{n}\right\|<\infty .
\end{gathered}
$$

For any $T \in L\left(H_{2}\right)$

$$
f(T)=\overline{g\left(S^{-1} T S\right)}=\sum_{n=1}^{\infty} \overline{\left(S^{-1} T S x_{n}, y_{n}\right)}
$$




$$
\begin{aligned}
& =\sum_{n=1}^{\infty} \overline{\left(\left(S^{-1}\right)^{*} y_{n}, T S x_{n}\right)} \\
& =\sum_{n=1}^{\infty}\left(T S x_{n},\left(S^{*}\right)^{-1} y_{n}\right) .
\end{aligned}
$$

$$
f=\sum_{n=}^{\infty}\left(u_{n}, \otimes v_{n}\right)
$$

and $\left(u_{n} \otimes v_{n}\right) \in(\operatorname{Alg} \phi(\mathscr{F})) \perp, \sum_{n=1}^{\infty}\left\|u_{n}\right\|\left\|v_{n}\right\|<\infty . \quad$ And therefore Alg $\phi(\mathscr{F})$ is hyperreflexive by

Theorem A. The proof is complete.

A PROOF OF THEOREM 2. Since $0 \bar{\epsilon} \pi(A), R(A)$, the range of $A$, is a closed subspace of $H$. Let $H_{1}=H(A)$, then $\phi_{A}$ defines a lattice isomorphism from $\xi(H)$ onto $\xi\left(H_{1}\right)$. From Lemma 1 we have that $\operatorname{Alg}_{H_{1}} \phi_{A}(\mathcal{F})=\left\{T \in L\left(H_{1}\right): \phi_{A}(\mathcal{F}) \subseteq \operatorname{Lat}(T)\right.$ is hyper-reflexive. By the definition of hyper-reflexivity, there exists $K>0$ such that for any $T \in L\left(H_{1}\right)$

$$
\begin{aligned}
& \operatorname{dist}\left(T, \operatorname{Alg}_{H_{1} \phi^{\phi}}(\text { F) })\right. \\
& \leq K \sup \left\{\left\|\left(I_{H_{1}}-\bar{P}_{\phi_{A}(M)}\right) T \bar{P}_{\phi_{A}(M)}\right\|: M \in \mathcal{F}\right\}
\end{aligned}
$$

where $P_{\phi}(M)$ denotes the orthogonal projection from $H_{1}$ onto $\phi_{A}(M)$. For any $T \in \operatorname{Alg}_{H_{1}}$ $\left(\phi_{A}(\mathscr{F})\right)$ and $S \in L(H)$, we define an operator $\widetilde{T} \in L(H)$ by formula

$$
\widetilde{T}(x \otimes y)=T x+S y, \quad x \otimes y H=H_{1} \otimes H_{1}^{\perp}
$$

Then $\tilde{T} \in \operatorname{Alg}\left(\phi_{A}(\mathscr{F}) \cup\{H\}\right)$ since $T \in \operatorname{Alg}_{H_{1}} \phi_{A}(\mathscr{F})$. Denote by $E$ the orthogonal projection from $H$ onto $H_{1}$, then

and so

$$
\begin{aligned}
& \operatorname{dist}\left(S, \operatorname{Alg}\left(\phi_{A}(\mathscr{F}) \cup\{H\}\right)\right) \\
& \leq\|S-T\| \\
& \leq\left\|\left.E S\right|_{H_{1}}-T\right\|+\left\|E^{\perp} S E\right\|
\end{aligned}
$$

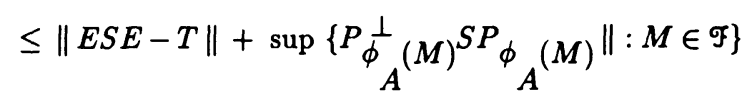

$$
\begin{aligned}
& \operatorname{dist}\left(S, \operatorname{Alg}\left(\phi_{A}(\boldsymbol{F}) \cup\{H\}\right.\right. \\
& \leq \operatorname{dist}\left(E S E, \operatorname{Alg} H_{1} \phi_{A}(\mathcal{F})\right)+\sup \left\{\left\|P_{\phi_{A}}^{\perp}(M)^{S P_{\phi}}(M)\right\|: M \in \mathcal{F}\right\} \\
& \leq(K+1) \sup \left\{\left\|P_{\frac{1}{N}}^{\frac{1}{}} S P_{N}\right\|: N \in \phi_{A^{(\mathscr{F})} \cup\{H\}}\right\}
\end{aligned}
$$

which implies the hyper-reflexivity of $\operatorname{Alg}_{A}(\Im) \cup(H\}$. Together with the reflexivity of $\phi_{A}(\Im) \cup\{H\}$ 
(see [3]), we obtain $\phi_{A}(\mathscr{F}) \cup\{H\}$ is hyper-reflexivity

\section{REFERENCES}

1. FILLMORE, P.A. and LONGSTAFF, W.E., On isomorphism of lattices of closed subspaces, Cand. J. Math., 36 (1984), 820-829.

2. HARRISON, K.J. and LONGSTAFF, W.E., Automorphic images of commutative subspace lattices, Trans. Amer. Math. Soc., $\underline{296}$ (1986), 217-228.

3. LONGSTAFF, W.E., Subspaces maps of operators on Hilbert spaces, Proc. Amer. Math. Soc., 84 (1982), 195-201.

4. ARVESON, W., Ten lectures on operator algebras. CBMS Regional Conference Series, No. 55, A.M.S., Providence, 1984.

5. KRAUS, J. and LARSON, D.R., Reflexivity and distance formulae, Proc. London Math. Soc., 53 (1986), 340-356. 


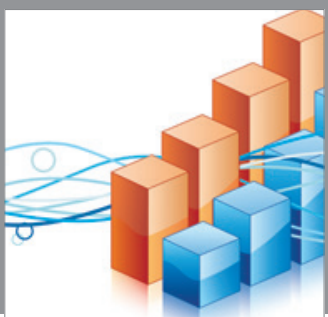

Advances in

Operations Research

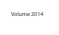

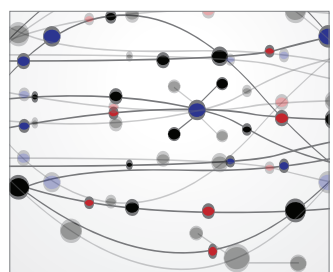

\section{The Scientific} World Journal
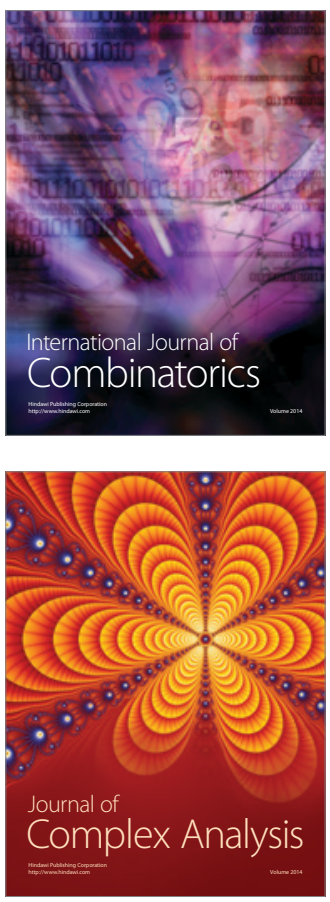

International Journal of

Mathematics and

Mathematical

Sciences
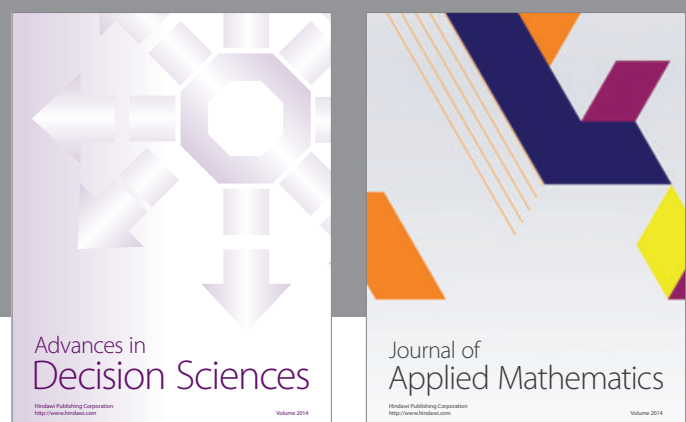

Journal of

Applied Mathematics
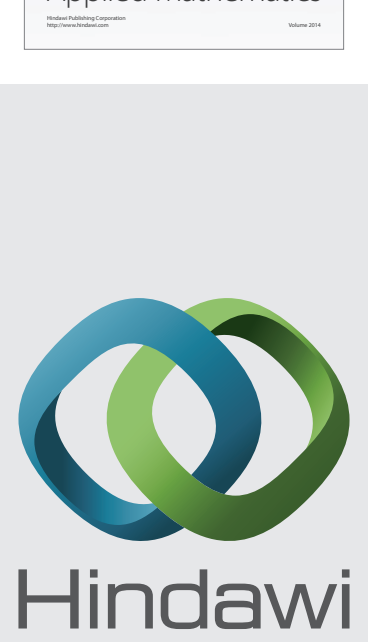

Submit your manuscripts at http://www.hindawi.com
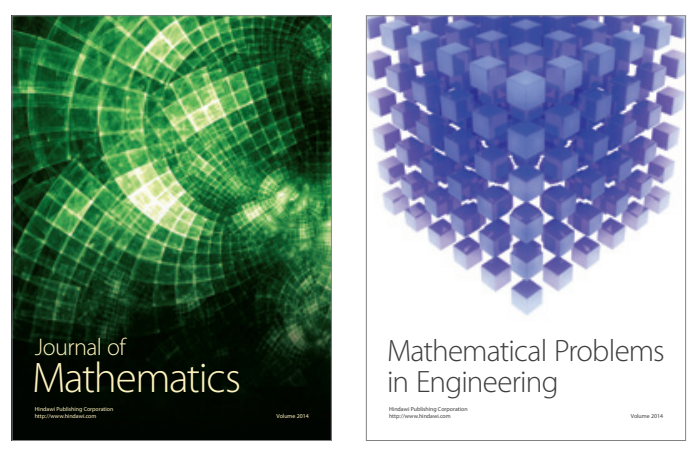

Mathematical Problems in Engineering
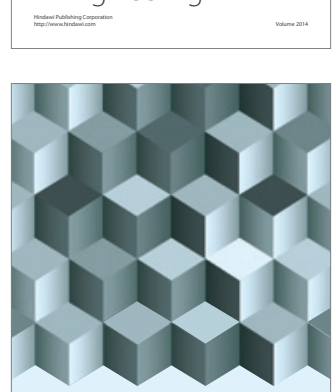

Journal of

Function Spaces
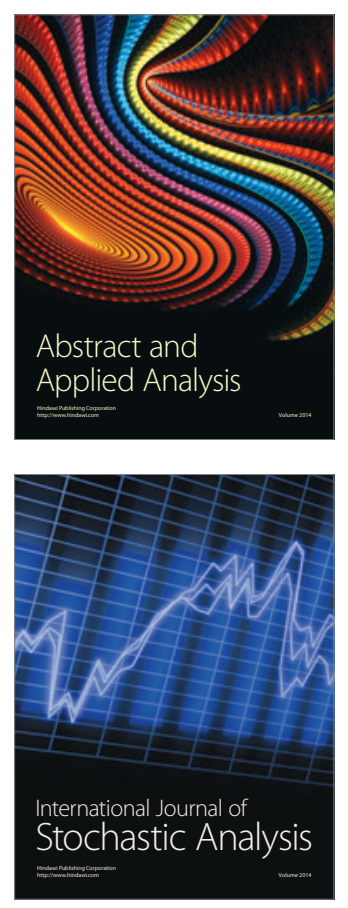

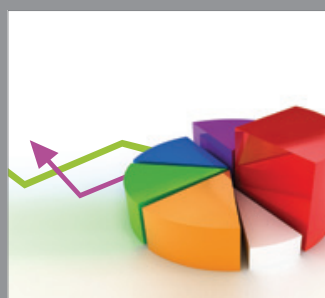

ournal of

Probability and Statistics

Promensencen
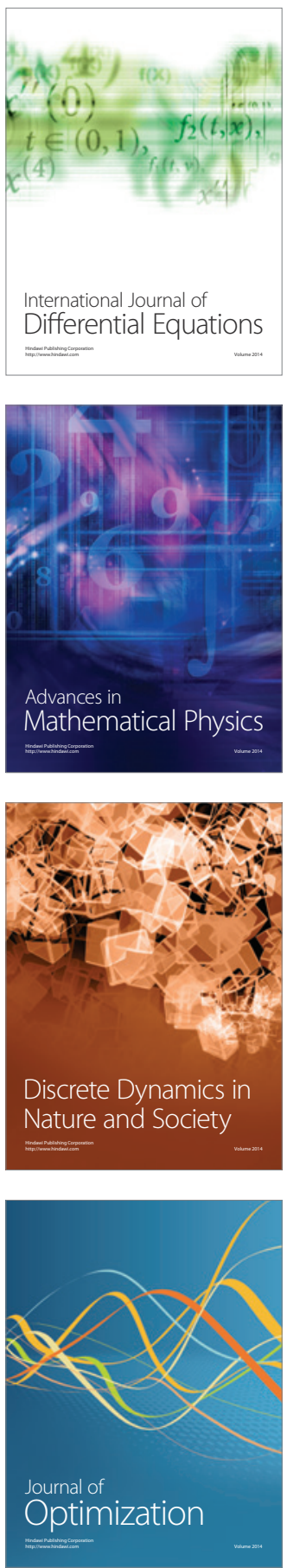